\title{
Les Perforations D'ulcere Gastro-Duodenal Au Centre National Hospitalier Et Universitaire (Cnhu) De Cotonou (Benin)
}

\author{
Vignon $\mathrm{Kc}$ \\ Mehinto Dk \\ Clinique Universitaire de Chirurgie Viscérale « B »- CNHU-HKM \\ Vignon $\mathrm{Kr}$ \\ Service d'Hépato gastroentérologie de l'Hôpital \\ d'Instruction des Armées de Cotonou \\ Mbele Rii \\ Service de Chirurgie Générale de l'Hôpital de District \\ de Biyem-Assi de Yaoundé (Cameroun)

\section{Natta N'tcha Nh \\ Hounkpe Ej} \\ Clinique Universitaire de Chirurgie Viscérale « B » - CNHU-HKM \\ CNHU-HKM: Centre National Hospitalier et Universitaire-Hubert \\ Koutoukou Maga. Cotonou-Bénin.
}

\section{doi: 10.19044/esj.2016.v12n27p117 URL:http://dx.doi.org/10.19044/esj.2016.v12n27p117}

\begin{abstract}
Abstact
Introduction: Duodenal or gastric ulcer perforations constitute a medical and surgical emergency.

Purpose: Expose its epidemiological, diagnostic and therapeutic aspects. Method: About a retrospective study from January 1st 2006 to March 31st, 2012 in visceral surgery at CNHU of Cotonou, records of 86 cases of duodenal or gastric ulcer perforation were analyzed.

Results: On average 13.8 cases / year, perforation of peptic ulcer was the most etiological cause of acute generalized peritonitis $(25.7 \%)$. The average age was 34.2 years, the sex ratio of 16.2. Average admission stay was 6 days and it was observed: undocumented epigastralgia (42cas; 48.8\%), no pathological medical history (32 cases; $37.2 \%$ ). Peritoneal syndrome was in 81 cases $(94.2 \%)$. Abdomen radiography without preparation (71 patients / $86 ; 82.6 \%$ ) showed a pneumoperitoneum (52 cases / 71; 77.6\%). The treatment was nonoperative ( 2 cases / 86; $2.3 \%$ ) with a favorable outcome. Surgical patients had duodenal perforation (49 cases / 84;57\%), gastric perforation $(35$ cases / 84; 43\%). Suturing with or without other technical
\end{abstract}


devices was achieved in all cases. Postoperative complications were present in $28.5 \%$ of cases and parietal abscesses were documented as a priority (14 cases / 84; 16.7\%). Mortality rate was $4.7 \%$ (4/86) and the average hospital stay was 11.6 days.

Conclusion: The perforations of peptic ulcer were common in young men. Clinical examination was especially helpful in the diagnosis by abdomen radiography without preparation. The treatment was medical and surgical and the outcome was mostly favorable.

Keywords: Peptic ulcer, perforation, suture

\section{Résumé:}

Introduction: Les perforations d'ulcère gastrique ou duodénale constituent une urgence médico-chirurgicale.

But: Exposer ses aspects épidémiologiques, diagnostiques et thérapeutiques.

Méthode: A partir d'une étude rétrospective du $1^{\mathrm{er}}$ Janvier 2006 au 31 Mars 2012 en chirurgie viscérale au CNHU de Cotonou, les dossiers de 86 cas de perforation d'ulcère gastrique ou duodénale ont été analysés.

Résultats: En moyenne 13,8 cas/an, la perforation d'ulcère gastro-duodénal était l'étiologie la plus fréquente des péritonites aiguës généralisées $(25,7 \%)$. L'âge moyen était de 34,2 ans, la sex-ratio de 16,2. Le délai moyen d'admission était de 6 jours et on notait: épigastralgie non documentée (42cas; 48,8\%), aucun antécédent médical pathologique (32 cas; 37,2\%). Le syndrome péritonéal était présent dans 81 cas $(94,2 \%)$. La radiographie de l'abdomen sans préparation (71 patients/86; 82,6\%) montrait un pneumopéritoine $(52 \mathrm{cas} / 71 ; 77,6 \%)$. Le traitement était non opératoire (2 cas $/ 86 ; 2,3 \%$ ) avec une évolution favorable. Les opérés présentaient une perforation duodénale (49 cas/84; 57\%), une perforation gastrique (35 cas $/ 84 ; 43 \%)$. Une suture associée ou non à d'autres artifices techniques était réalisée dans tous les cas. Les complications postopératoires $(28,5 \%)$ étaient dominées par les suppurations pariétales (14 cas/84; 16,7\%). La mortalité était de 4,7\% (4/86) et la durée moyenne d'hospitalisation de 11,6 jours.

Conclusion: Les perforations d'ulcère gastro-duodénal étaient fréquentes chez l'homme jeune. Le diagnostic clinique était surtout aidé par la radiographie de l'abdomen sans préparation. Le traitement a été médicochirurgical et l'évolution était surtout favorable.

Mots-clés: Ulcère gastroduodénal, perforation, suture

\section{Introduction}

La perforation complique 2 à $11 \%$ des ulcères gastroduodénaux et affecte sans prédominance d'un sexe des sujets adultes jeunes souvent 
défavorisés (Casali 2012, Moller 2009). Elle est grave et siège sur le duodénum dans $60 \%$ des cas et sur l'estomac dans $40 \%$ des cas. Ses 2 principales causes sont l'infection à Helicobacter pylori et la prise d'antiinflammatoires non stéroïdiens (Casali 2012). Cette pathologie est souvent révélée par un abdomen aigu et l'imagerie notamment la radiographie de l'abdomen sans préparation mais surtout la tomodensitométrie aide au diagnostic (Amela 2006). Son traitement est médico-chirurgical. Sa prévention ou la réduction de sa prévalence viendra de l'amélioration du traitement d'éradication de l'Helicobacter pylori (Malfertheiner 2007). Notre but a été d'exposer les aspects épidémiologiques, diagnostiques et thérapeutiques des cas de perforation d'ulcère gastrique ou duodénale dans une population à condition socio-économique modeste.

\section{Méthode}

Il s'agissait d'une étude rétrospective du $1^{\text {er }}$ janvier 2006 au 31 Mars 2012 dans les services de chirurgie viscérale " $A$ " et 'B" du CNHU-HKM de Cotonou. Elle a porté sur les dossiers des patients hospitalisés pour perforation d'ulcère gastrique ou duodénale. Nos critères d'inclusion ont été l'existence:

-d'une observation médicale complète,

-pour les patients non opérés, l'existence d'une fibroscopie oesogastro-duodénale antérieure attestant le diagnostic d'ulcère

-pour les patients opérés, l'existence d'un compte rendu opératoire, la biopsie de toutes les perforations gastriques confirmant le diagnostic d'ulcère.

Les variables étudiées ont été : âge, sexe, profession, mode et délai d'admission, motif de consultation, antécédents médicaux, signes cliniques, signes paracliniques, diagnostic étiologique préopératoire, traitement, durée d'hospitalisation, évolution.

Le diagnostic clinique était surtout aidé par la radiographie de l'abdomen sans préparation à défaut du scanner qui est le plus important examen d'imagerie dans cette pathologie (Amela 2006)

\section{Résultats}

\section{Aspects épidémiologiques}

-Fréquence

Pendant la période d'étude, sur les 296 cas de péritonite aiguë généralisée pris en charge dans les cliniques universitaires de chirurgie viscérale " $A$ " et 'B', les perforations d'ulcère gastrique ou duodénal (86 cas; 29\%) étaient l'étiologie la plus fréquente. Elles étaient suivies des péritonites: appendiculaire ( 76 cas; $25,7 \%$ ), typhique ( 65 cas; $22 \%$ ), génitale (24 cas; $8,1 \%$ ), primitive (17 cas; 5,74\%), perforation traumatique du grêle 
(13 cas; 4,4\%), biliaire ( 7 cas; $2,5 \%$ ), perforation de tumeur digestive ( 4 cas; $1,3 \%)$, rupture d'abcès hépatique ( 3 cas; $1 \%$ ) et perforation de vessie nécrosée sur sonde vésicale chez un paraplégique (1 cas; 0,3\%). La fréquence des péritonites par perforation d'ulcère gastrique ou duodénale a été de 13,8 cas/an.

-Age

L'âge moyen a été de 34,2 ans avec des extrêmes de 16 et 72 ans.

-Sexe

On avait dénombré 81 hommes et 5 femmes, soit une sex-ratio de 16,2 .

-Profession

On avait dénombré: fonctionnaire ( 32 cas; $37,2 \%)$, artisan ( 25 cas; $29,1 \%$ ), élève/étudiant (12 cas; 14\%), , ménagère, chauffeur, commerçant (5 dans chaque cas; $5,8 \%$ ), conducteur de taxi moto ( 2 cas; $2,3 \%$ ).

\section{Aspects diagnostiques}

\section{Mode d'admission}

Sur les 86 patients, 50 soit $58,1 \%$ étaient évacués d'un centre de santé périphérique et les 36 autres soit $41,9 \%$ venaient directement de leur domicile.

\section{Délai d'admission} jours.

Il était en moyenne de 6 jours avec des extrêmes de 6 heures et 21

\section{Motif de consultation}

Les motifs de consultation ont été: douleur abdominale généralisée (86 cas; 100\%), référence pour péritonite aiguë généralisée (26 cas; 30,2\%); arrêt des matières et des gaz ( 22 cas; $25,6 \%$ ), vomissement (49 cas; $57 \%$ ), diarrhée ( 3 cas; $3,5 \%)$.

\section{Facteurs déclenchants}

Ils ont été: prise d'antiinflammatoires non stéroïdiens (21 cas; 24,4\%), jeûne (13 cas; 15,1\%), alcoolisme (6 cas; 7\%). Dans 46 cas soit $53,5 \%$, aucun facteur déclenchant n'a été retrouvé.

\section{Siège initial de la douleur}

La figure $\mathrm{N}^{\circ} \mathbf{1}$ présente la répartition en fonction du siège initial de la douleur abdominale des patients pris en charge pour perforation d'ulcère gastrique ou duodénal. 


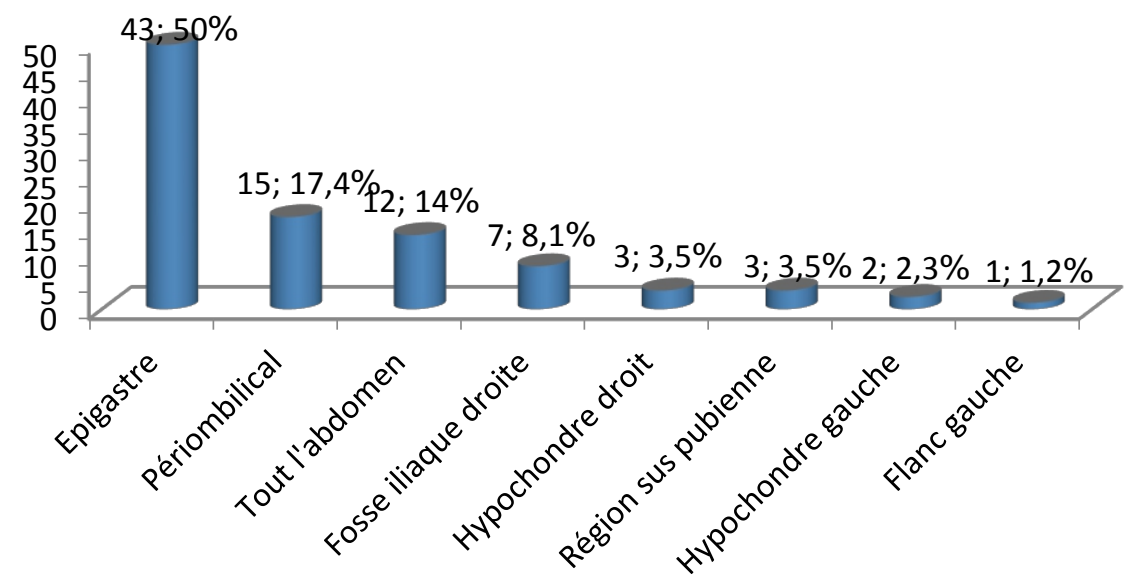

$\underline{\text { Figure } 1}$ : Répartition des patients pris en charge pour perforation d'ulcère gastrique ou duodénale en fonction du siège initial de la douleur abdominale

\section{Antécédents médicaux}

La répartition en fonction des antécédents médicaux des 86 patients atteints de perforation d'ulcère gastrique ou duodénal était: aucun antécédent médical pathologique (32 cas; $37,2 \%$ ); épigastralgie non explorée ( 28 cas; $32,6 \%$ ); ulcère gastrique confirmé par la fibroscopie œsogastroduodénale (12 cas; $14 \%$ ); ulcère duodénal confirmé par la fibroscopie œsogastroduodénale (7 cas; 8,1\%); hypertension artérielle (4 cas; 4,6\%); diabète, asthme, cardiopathie ischémique, drépanocytose SS, maladie de Parkinson (1 dans chaque cas; $1,2 \%$ )

\section{Etat général}

Il était bon chez 32 patients soit $37,2 \%$ et mauvais chez 54 patients soit $62,8 \%$

\section{Signes physiques}

Ils sont résumés dans le tableau I suivant.

Tableau I : Répartition des patients pris en charge pour perforation d'ulcère gastrique ou duodénale selon les signes physiques abdominaux

\begin{tabular}{ccc}
\hline Signes physiques & Nombre & Pourcentage \\
\hline Immobilité respiratoire de l'abdomen & 33 & $38,4 \%$ \\
Cri de l'ombilic & 74 & $86,1 \%$ \\
Défense abdominale & 53 & $61,6 \%$ \\
Contracture de l'abdomen & 21 & $24,4 \%$ \\
Absence de contracture ou de défense & 12 & $13,9 \%$ \\
Matité déclive des flancs & 31 & $36 \%$ \\
Disparition de la matité hépatique & 36 & $41,8 \%$ \\
remplacée par une sonorité préhépatique & & $81,4 \%$ \\
Cri de Douglas & 70 & \\
\hline
\end{tabular}




\section{Examens paracliniques}

-La radiographie de l'abdomen sans préparation (ASP)

Elle a été réalisée chez 71 patients soit 82,6\% et non faite chez 15 patients soit 17,4\%. Elle a été jugée de mauvaise qualité dans 3 cas soit $3,5 \%$, normale dans 1 cas soit $1,6 \%$ et anormales dans 67 cas soit $78 \%$. Les résultats de l'ASP des 67 patients étaient: croissant gazeux sous diaphragmatique (52 cas; 77,6\%); grisaille diffuse (33 cas; 49,3\%); images hydro-aériques ( 7 cas: 10,4\%); aérocolie ( 1 cas; 1,5\%).

-L'ionogramme sanguin et le bilan rénal réalisés chez tous les patients avaient montré: hyponatrémie entre 126 et $132 \mathrm{mEq} / 1$ (35 cas; 40,7\%); hypokaliémie entre 2,1 et $3,2 \mathrm{mEq} / 1$ (14 cas; 16,3\%); hypernatrémie à $152 \mathrm{mEq} / 1$ ( 1 cas; $1,2 \%)$; insuffisance rénale aiguë avec créatininémie élevée entre 16 et $80 \mathrm{mg} / 1$ (32 cas; 37,2\%).

\section{Diagnostic étiologique préopératoire}

Les diagnostics étiologiques préopératoires évoqués étaient: perforation d'ulcère gastrique ou duodénal (62 cas; 72,1\%); perforation d'organe creux sans précision de l'organe (11 cas; 12,8\%); origine appendiculaire (6 cas; 7\%); aucune étiologie évoquée (7 cas; 8,1\%).

\section{Aspects thérapeutiques}

\section{Traitement médical}

-Tous les patients avaient bénéficié d'une réanimation médicale

-L'utilisation d'association d'antibiotiques a été systématique chez tous les patients et était faite de: ceftriaxone + métronidazole (41 patients; 47,7\%); ciprofloxacine + métronidazole (19 patients; 22,1\%); amoxicilline + Métronidazole (13 patients; 15,1\%); amoxicilline + acide clavulanique + métronidazole (8 patients; 9,3\%); Amoxicilline + métronidazole + clarithromycine (5 patients; $5,8 \%$ ).

-Le traitement antiulcéreux

Il a été administré dans tous les cas et était fait de: Ranitidine (62 cas; 72,1\%); lanzoprazole (21 cas; 24,4\%); oméprazole ( 3 cas; 3,5\%).

\section{Traitement non opératoire (méthode de Taylor: aspiration gastrique} continue, sonde vésicale, antiulcéreux, association d'antibiotiques)

Il a été réalisé chez 2 patients ayant un antécédent d'épigastralgie, en bon état général et qui étaient admis respectivement 4 heures et 6 heures après le début de la symptomatologie. Tous présentaient à l'ASP un croissant gazeux sous diaphragmatique. 


\section{Traitement chirurgical}

-Une anesthésie générale et une laparotomie médiane ont été réalisées chez les 84 patients restants.

-La perforation a été duodénale chez 49 patients sur $84(57 \%)$ et gastrique chez 35 patients sur 84 (43\%). Toutes les perforations duodénales ont été bulbaires. Sur l'estomac, la perforation était sur: l'antre (21 cas/35; $60 \%)$, la petite courbure $(13$ cas/35; 37,1\%), le corps ( 1 cas/35; $2,9 \%)$.

-Actes posés

- Les 49 cas de perforation d'ulcère duodénal ont été traités par: excision des berges de la perforation + suture duodénale en pyloroplastie (49 cas; $100 \%$ ), omentoplastie (3 cas; $6,1 \%)$, vagotomie tronculaire bilatérale (31 cas; $63,2 \%$ ).

- Les 35 cas de péritonite par perforation d'ulcère gastrique ont été traités par: excision des berges de la perforation + suture (35 cas; $100 \%)$, omentoplastie ( 2 cas; $5,7 \%$ ), vagotomie tronculaire bilatérale ( 2 cas; $5,7 \%$ ). Ici un examen anatomopathologique du prélèvement des berges de la perforation, réalisé dans tous les cas, a confirmé le diagnostic et éliminer une lésion maligne.

La durée moyenne d'hospitalisation a été de 11,6 jours avec des extrêmes de 3 et 49 jours.

\section{Evolution}

\section{En cours d'hospitalisation}

-Pour les 2 patients ayant reçu un traitement non opératoire, l'évolution était favorable.

-Pour les 84 autres patients opérés, l'évolution était d'emblée favorable (56 cas/84; 66,7\%) et secondairement favorable (24 cas/84; $28,5 \%)$.

Les complications post opératoires étaient: suppuration pariétale (14 cas/84; 16,7\%), péritonite postopératoire (3 cas/84; 3,6\%), fistule stercorale (4 cas/84; 4,7\%), pneumopathie de décubitus (4 cas/84; 4,7\%), éviscération (3 cas/84; 3,6\%), anémie ( 2 cas/84; $2,4 \%)$.

-Nous avions dénombré 4 décès $(4,7 \%)$ sur les 86 patients pris en charge pour perforation d'ulcère gastrique ou duodénale. Les causes de décès ont été: état de choc hypovolémique ( 2 cas), détresse respiratoire (1 cas), accident vasculaire cérébral sur poussée d'hypertension artérielle

\section{En dehors de l'hospitalisation}

Sur les 80 patients opérés et sortis de 1'hôpital, 2 soit 2,5\% avaient présenté une éventration à un recul de 6 mois et aucun décès de plus n'a été noté. 


\section{Discussion}

\section{Epidémiologie}

-Fréquence

Dans notre étude, sur les 296 cas de péritonite aiguë généralisée recensés, ceux dus à une perforation d'ulcère gastrique ou duodénale étaient majoritaires et représentent 29\%. Cependant (Harouna 2001) au Niger avait noté que les perforations d'ulcère gastrique ou duodénal occupaient le troisième rang des causes de péritonite après celles d'origine appendiculaire et par perforation iléale typhique avec un taux de $15 \%$. Dans notre série, la fréquence des perforations d'ulcère gastrique ou duodénal était en moyenne de 13,8 cas/an. Notre fréquence moyenne est inférieure à celles égales à 16,5 cas/an et 17 cas/an observées respectivement par (Schein M 1986) et (Chalya 2011). Ce constat pourrait s'expliquer par le fait que la population béninoise prend de plus en plus conscience des complications liées à la pathologie ulcéreuse dont le traitement devient progressivement accessible. -Age

L'âge moyen dans notre étude était de 34,2 ans. Il s'agit donc en majorité d'une population jeune. Ce constat avait été fait par plusieurs auteurs (Jordan P H Jr 1995, L'helgouarc'h 2000, Rahuman 2003) qui ont noté dans leurs séries que l'’âge moyen variait entre 35 et 47 ans. Par contre, cette moyenne d'âge était plus élevée dans d'autres séries (Moller 2009, Kang 2006) notamment des pays développés et était respectivement égale à 56 ans et 60 ans.

-Sexe

Une prédominance masculine a été notée $(81$ hommes contre 5 femmes) dans notre travail, comme dans d'autres études (Diallo 2007, Ohene-Yeboah 2006). Nos résultats sont différents de ceux obtenus par d'autres auteurs (Moller 2009, Tessema 2005) qui notent que cette pathologie intéresse de façon équivalente les hommes et les femmes.

\section{Aspects diagnostiques}

\section{Aspects cliniques}

-Facteurs déclenchants

Dans notre série, ont été incriminés les antiinflammatoires non stéroïdiens ( 21 cas; $24,4 \%$ ), le jeûne (13 cas; 15,1\%), l'alcoolisme (6 cas; $7 \%$ ). Plusieurs facteurs plus ou moins associés tels que les antiinflammatoires non stéroïdiens, le jeûne, le tabac ainsi que l'alcoolisme chronique semblent être incriminés dans la survenue de la perforation de l'ulcère gastroduodénal (L'helgouarc'h 2000, Ohene-Yeboah 2006).

-Comme dans d'autres études (Jeffrey 2001, Gonzalez 1997), le siège initial de la douleur était en majorité épigastrique (50\%) dans notre travail. -Antécédents médicaux 
Presque le tiers des patients (28 cas/86; 32,56\%) avaient un antécédent d'épigastralgie non documentée, $32(37,2 \%)$ n'avaient aucun antécédent médical pathologique et seuls $19(22,1 \%)$ avaient un ulcère gastroduodénal antérieurement confirmé à la fibroscopie. Ainsi le diagnostic étiologique avait été fait dans la majorité des cas en peropératoire. Des constats analogues avaient été rapportés par d'autres auteurs africains (Chalya 2011, Kang 2006, Nasio 2009) qui l'expliquent par la difficulté d'accès aux soins qui caractérise la grande partie des populations des pays en voie de développement.

-La majorité des patients de notre étude ( 54 cas/86; 62,8\%) avait un mauvais état général, certainement lié à un délai d'admission qui était souvent long (6 jours en moyenne). Le même constat avait été fait dans par plusieurs auteurs (Chalya 2011, Tessema 2005, Nasio 2009) qui l'attribuent à l'impossibilité d'accès à des soins coûteux pour des malades souvent démunis.

-Comme dans d'autres séries (Nasio 2009, Kuremu 2002), la radiographie de l'abdomen sans préparation nous a permis d'évoquer le diagnostic de perforation d'un viscère creux devant la présence d'un croissant gazeux sous diaphragmatique chez la plupart des patients (52 cas/71; 73,2\%). Aucun de nos patients n'a bénéficié d'une tomodensitométrie qui est aujourd'hui le « gold standard » dans le diagnostic des perforations de viscères creux montrant de petits pneumopéritoines qui passent inaperçus à l'ASP (Amela 2006).

\section{Aspects thérapeutiques}

-Traitement non opératoire

Il a été réalisé seulement chez 2 patients $(2,4 \%)$ dans notre étude car les critères de sa réalisation n'étaient pas présents chez les autres. L'évolution a été favorable dans les 2 cas. En effet, plusieurs auteurs rapportent qu'en utilisant ce moyen thérapeutique exigeant, l'on obtient $80 \%$ de réussite chez des patients de moins de 70 ans, en bon état général, pris en charge dans les 24 heures, à jeun au moment de la perforation et sans signes de gravité clinique ou biologique (Rahuman 2003, Mouly 2013).

-Traitement chirurgical

.Il reste le traitement de référence de l'ulcère gastroduodénal perforé (Mouly 2013) et il n'a pas échappé au développement de la coeliochirurgie. Cette dernière, en plus de ses avantages prouvés par rapport à la laparotomie que sont le moindre risque de complications pariétales, la meilleure tolérance respiratoire post-opératoire, une durée d'hospitalisation plus courte, une réduction des douleurs et une réhabilitation précoce; a un taux de succès de 92\% (Mouly 2013, Sanabria 2013). La coeliochirurgie n'a été réalisée chez aucun des patients de notre travail car ce type de chirurgie n'est pas 
développé dans notre milieu de travail à cause des contraintes matérielles et économiques qu'il implique.

.En peropératoire, Il a été observé que la perforation d'ulcère a été plus duodénale (49 patients/84; 57\%) que gastrique (35 patients/84; 43\%) avec un ratio ulcère duodénal sur ulcère gastrique égal à 1,4 . La prédominance des perforations d'ulcère duodénal a aussi été observée par d'autres auteurs avec un ratio nettement plus élevé que le nôtre. Il s'agit de (Chalya 2011, Kuremu 2002) qui avaient rapporté respectivement un ratio de 12,7 et 11,5. Par contre, (Ohene-Yeboah 2006) avait plutôt observé plus de perforation d'ulcère gastrique dans son étude avec un ratio égal à 0,4 .

.Les 49 cas de perforation d'ulcère duodénal ont été traités par: suture (100\% des cas), vagotomie tronculaire bilatérale (31 cas; 63,2\%) et omentoplastie (3 cas; 6,1\%). Pour certains auteurs (Mouly 2013, Ates 2007) le traitement de la perforation par suture associée à une omentoplastie ou l'application de colle biologique, utilisation isolée de patch de collagène, n'a pas montré sa supériorité par rapport à la suture simple. Depuis l'avènement et l'efficacité prouvée des inhibiteurs de la pompe à protons, et des antibiotiques d'éradication de l'Helicobacter pylori, l'association à la suture de la perforation, d'une vagotomie n'est plus recommandée (Mouly 2013, Gutiérrez de la Peña 2000).

.En cas de perforation d'ulcère gastrique la suture simple des berges avec ou sans omentoplastie reste la technique la plus utilisée (Mouly 2013); ce qui a été le cas dans notre étude.

-Complications post opératoires

Dans notre série, les complications étaient survenues chez 28,5\% des patients opérés et étaient dominées par les suppurations pariétales (16,7\%). Nos résultats sont comparables à ceux de (Chalya 2011) qui avait rapporté $29,8 \%$ de complications post opératoires dans son travail.

-La mortalité était de $4,7 \%$ et est nettement inférieure à celles de $10,7 \%$ et $22,1 \%$ rapportées respectivement par (Chalya 2011 et OheneYeboah 2006).

\section{Conclusion}

Les péritonites par perforation d'ulcère gastrique ou duodénal étaient fréquentes et surtout observées chez le sujet jeune de sexe masculin. La radiographie de l'abdomen sans préparation a aidé au diagnostic dans la majorité des cas. Le traitement a été médico-chirurgical et l'évolution était surtout favorable. 


\section{References:}

Casali JJ, Franzon O, Kruel NF, Neves BD. Epidemiological analysis and use of rapid urease test in patients with perforated peptic ulcers. Rev Col Bras Cir 2012; 39 (2): 93-8. Pub Med / Google Scholar.

Moller MH, Shah K, Bendix J, Jensen AG et al. Risk factors in patients surgically treated for peptic ulcer perforation. Scand J Gastroenterol 2009; 44 (2): 145-52. Pub Med / Google Scholar.

Amela S, Serif B, Lidija L. Early radiological diagnostics of gastrointestinal infection in the management of peptic ulcer perforation. Radiol Oncol 2006, 40 (2): 67-72.

Malfertheiner P, Megraud F, O'Morain C et al. Current concepts in the management of Helicobacter pylori infection: the Maastricht III Consensus report. Gut 2007; 56 (6): 772-81. Pub Med / Google Scholar-gut.bmj.com

Harouna Y, Ali L, Seibou A, Abdou I, Gamatie Y, Rakotomalala J, Habibou A, Bazira : Deux ans de chirurgie digestive d'urgence à l'hôpital National de Niamey ( Niger) Méd Afr 2001; 48 (2 ) : 49 53. Pub Med / Google Scholar-santetropical.com

Chalya PL, Mabula JB, Koy M, Mchembe MD, Jaka HM, Kabangila R et al. Clinical profile and outcome of surgical treatment of perforated peptic ulcers in Northwestern Tanzania: A tertiary hospital experience. World Journal of Emergency Surgery 2011, 6 (31): 1-10. Pub Med / Google Scholar.-wjes.biomedcentral.com

L'helgouarc'h J L, Feschand F, Benoit L, Goudet P, Couguard P. Traitement des ulcères duodénaux perforés par laparoscopie. 35 cas. La presse Médicale 2000; 29 (27): 84-9.

Rahuman MM, Saha A K, Rahim A. Experience of pectic ulcer perforation over a decade in a teaching hospital of Southern Bangladesh. Ceylon Med J 2003; 48 ( 2 ): 53 - 5. Pub Med / Google Scholar.

Kang JY, Elders A, Majeed A. Recent trend in hospital admission and mortality rate for peptic ulcer in Scotland 1982_2002. Aliment Pharmacol Ther 2006, 24 (1): 65-79.

Diallo AT, Touré A, Mehinto DK, Olory-Togbé JL, Touré FB, Camara ND. Perforation de l'ulcère duodénal: prise en charge thérapeutique au CHU Ignace Deen de Conakry. J Afr Chir Digest 2007; 7 (2): 697-703.

Ohene-Yeboah M, Togbe B. Perforated gastric and duodenal ulcers in an urban african population. West Afr J Med 2006; 25 (3): 205-11.

Tessema E, Meskel Y, Kotiss B. Perforated peptic ulcer in Tikur Anbessa Hospital. Ethiop Med Journal 2005, 43 (1): 9-13.Pub Med / Google Scholar tspace.library.utoronto.ca 
Jeffrey AN, Randal R, Alfred EC, Stephen FH, Robert WT. Surgery basic science and clinical evidence. USA: Donnelley and Sons, Willard OH; 2001: 489-500. Pub Med / Google Scholar.

Gonzalez N, Fourtanier G, Suc B. Complications chirurgicales des ulcères gastroduodénaux. EMC Gastroentérologie. 9-022-A10, 1997,6p.

Nasio NA, Saidi H. Perforated peptic ulcer disease at Kenyatta National Hospital, Nairobi. East and Central African Journal of Surgery 2009, 14 (1): 13-6. Pub Med / Google Scholar.

Kuremu RT. Surgical management of peptic ulcer disease. East Afr Med J 2002, 76 (9): 454-6. Pub Med / Google Scholar.

Mouly C, Chati R, Scotté M, Regimbeau J-M. Prise en charge de l'ulcère gastroduodénal perforé: revue de littérature. Journal de Chirurgie Viscérale 2013, 150: 356-64. Pub Med / Google Scholar

Sanabria AE, Villegas MI, Morales Uribe CH. Laparoscopic repair for perforated peptic ulcer disease (review). Cochrane Database Syst Rev 2013; 28 (2): CD004778. Pub Med / Google Scholar.

Ates M, Sevil S, Bakircioglu E, Colak C. Laparoscopic repair of peptic ulcer perforation without omental patch versus conventional open repair. J Laparoendosc Adv Surg Tech A 2007; 17 (5): 615-9.Pub Med / Google Scholar.

Gutiérrez de la Peña C, Marquez R, Fakih F, Dominguez-Adame E, Medina J. Simple closure or vagotomy and pyloroplasty for the treatment of a perforated duodenal ulcer: Comparison of results. Dig Surg 2000; 17 (3): 225-8. Pub med / Google Scholar. 